\title{
Buckling-Induced Reversible Symmetry Breaking and Amplification of Chirality Using Supported Cellular Structures
}

\section{Citation}

Kang, Sung Hoon, Sicong Shan, Wim L. Noorduin, Mughees Khan, Joanna Aizenberg, and Katia Bertoldi. 2013. "Buckling-Induced Reversible Symmetry Breaking and Amplification of Chirality Using Supported Cellular Structures." Advanced Materials 25 (24) (May 2): 3380-3385. doi:10.1002/adma.201300617.

\section{Published Version}

doi:10.1002/adma.201300617

\section{Permanent link}

http://nrs.harvard.edu/urn-3:HUL.InstRepos:37255494

\section{Terms of Use}

This article was downloaded from Harvard University's DASH repository, and is made available under the terms and conditions applicable to Open Access Policy Articles, as set forth at http:// nrs.harvard.edu/urn-3:HUL.InstRepos:dash.current.terms-of-use\#OAP

\section{Share Your Story}

The Harvard community has made this article openly available.

Please share how this access benefits you. Submit a story. 
DOI: $10.1002 / \mathrm{adma} .201300617$

\section{Submitted to

\section{Buckling-Induced Reversible Symmetry Breaking and Amplification of Chirality Using Supported Cellular Structures}

By Sung Hoon Kang ${ }^{1 \dagger}$, Sicong Shan ${ }^{1 \dagger}$, Wim L. Noorduin ${ }^{1 \dagger}$, Mughees Khan ${ }^{2}$, Joanna Aizenberg ${ }^{1,2,3 *}$, and Katia Bertoldi ${ }^{1,3 *}$

[*] Prof. J. Aizenberg and Prof. K. Bertoldi, Dr. S. H. Kang, S. Shan, Dr. W. L. Noorduin 1 School of Engineering and Applied Sciences, Harvard University

Cambridge, MA 02138 (USA)

E-mail: jaiz@seas.harvard.edu (Prof. J. Aizenberg) and bertoldi@seas.harvard.edu (Prof. K. Bertoldi)

$\dagger$ These authors contributed equally to this work.

Dr. M. Khan

2 Wyss Institute for Biologically Inspired Engineering, Harvard University

Boston, MA 02115 (USA)

Prof. J. Aizenberg and Prof. K. Bertoldi

3 Kavli Institute for Bionano Science and Technology, Harvard University

Cambridge, MA 02138 (USA)

Keywords: buckling, chirality, handedness, reversibility, cellular structures

Robust routes to induce chirality in centrosymmetric systems are not only of fundamental interest in unraveling the origin of single chirality as found in nature, but also have a broad range of practical applications including optical devices, ${ }^{[1-3]}$ sensors, ${ }^{[4]}$ pharmaceuticals, ${ }^{[5]}$ and advanced structural components. ${ }^{[6]}$ Since many chiral systems show distinctive properties depending on their handedness, ${ }^{[4,7-8]}$ elucidating routes to induce chiral symmetry breaking and to control the handedness across different length scales is crucial. In general, the emergence of chiral structures of single handedness relies on a two-step process: (i) spontaneous symmetry breaking followed by (ii) full amplification of this chiral imbalance to yield a uniform configuration. On the molecular scale, experimental demonstrations of such processes have been reported only for few crystallizing systems and chemical reactions, ${ }^{[9-12]}$ in which the amplification relies on activating and inhibiting interactions at the molecular scale. ${ }^{[13]}$ Extending these principles to larger length scales requires fundamentally different concepts. Although centrosymmetric nanopost arrays ${ }^{[14-16]}$ and swellable confined nanoscale gel films ${ }^{[17-18]}$ were recently reported to form chiral structures in the absence of chiral 
aneous and reversible chiral symmetry induction, no study has been reported on the spontaneous and reversible chiral symmetry breaking and subsequent full amplification to yield large areas of ordered structures with single handedness.

Guided by a theoretical model, we here exploit buckling in rationally designed supported cellular structures at the mesoscale to reversibly switch between the initially achiral configuration and subsequent uniform left- or right- handed configurations. Moreover, we demonstrate that the underlying principles can be generalized using different geometries, materials, stimuli, and length scales. Although buckling is often regarded as a failure mode to avoid in structural materials, there are numerous natural ${ }^{[19-20]}$ and artificial ${ }^{[21-28]}$ systems that use it to their advantage. Buckling of surface-attached strips ${ }^{[29]}$ and free-standing structures ${ }^{[30]}$ has been studied extensively, but little has been known about mechanical instabilities in surface-attached cellular structures. Moreover, while the deformation and unique mechanical properties of chiral cellular structures have been the focus of many studies, ${ }^{[1-32]}$ here we demonstrate spontaneous and reversible chiral symmetry breaking in initially achiral structures followed by full amplification of the chiral imbalance across the entire structure to yield configurations of single handedness.

We start by recognizing that the formation of a chiral pattern, in which all vertices rotate in the same direction, requires that all the individual plates buckle in a higher mode comprising an integer number of complete sinusoids, as shown in Figure 1 (a, bottom) for a honeycomb as a model cellular structure. Therefore, simply buckling a free-standing honeycomb structure is not sufficient to form a chiral structure, since this induces a first-order mode in all plates, ${ }^{[30,33]}$ leading to an achiral configuration where adjacent vertices alternately rotate clockwise and counterclockwise, as shown in Figure 1(a, top). However, the attachment of the cellular structure to a rigid substrate allows higher order buckling modes, so that one can design architectures capable of deforming into either achiral or chiral configurations. 


\section{Submitted to

Based on elastic plate theory, we anticipate that the buckling mode can be controlled by carefully designing the dimensions of the cellular architecture. Furthermore, we expect that the connectivity of these cellular structures contributes to the uniform spreading of the chiral pattern once the symmetry is locally broken.

To define the design criteria and predict the deformation of the ensemble, we conducted elastic buckling analysis of an individual supported thin plate. Assuming that the thin plate of length $l$, height $h$, and thickness $t$ may be described as a linear elastic material with Young's modulus $E$, Poisson's ratio $v$, and bending stiffness $D=E t^{3} /\left[12\left(1-v^{2}\right)\right]$, its buckling behavior is described by ${ }^{[34]}$

$$
\frac{\partial^{4} w}{\partial x^{4}}+2 \frac{\partial^{4} w}{\partial x^{2} \partial y^{2}}+\frac{\partial^{4} w}{\partial y^{4}}+\frac{E t \varepsilon_{s w}}{D} \frac{\partial^{2} w}{\partial x^{2}}=0
$$

where $w$ is the deflection in the out-of-plane direction and $\varepsilon_{s w}$ denotes the differential swelling strain which is assumed to be uniform within the plate. To consider the effect of the neighboring plates in the lattice structures, we assume the two vertical edges at $x=0$ and $x=l$ to be supported (i.e. $w=0$ and $\frac{\partial^{2} w}{\partial x^{2}}+v \frac{\partial^{2} w}{\partial y^{2}}=0$ ), so that the solution of Eq. (1) has the form $w=f(y) \sin \frac{m \pi x}{l}, m$ being an integer (see Supporting Information). For a plate attached to a rigid substrate (i.e. free at $\mathrm{y}=h$ and clamped at $y=0$ ), the critical swelling strain $\varepsilon_{s w}^{c r}$ and the wavelength $2 \mathrm{l} / \mathrm{m}$ of the corresponding mode can then be solved as a function of the aspect ratio $l / h$, as shown in Figure $1(\mathrm{~b})$. This stability diagram unambiguously reveals that in an initially straight plate with $0<l / h<2.321$ a buckling pattern with half sinusoidal wavelength (i.e. $m=1$ ) will emerge upon reaching a critical swelling strain, resulting in an achiral pattern (space group: P31m). Differently, if $2.321<l / h<4.015$ the buckling will be a full sinusoid (i.e. $m=2$ ), yielding a chiral pattern (space group: P6). To fully define the geometry of supported 
cellular structure for experiments, we investigated the effect of the thickness of the plates using non-linear finite element analyses (see Supplementary Methods and Supplementary Figures S1 and S2). These numerical calculations show that the thickness only marginally affects the buckling modes (Supplementary Figure S2), confirming that the buckling-induced pattern of supported cellular structures is dictated by the aspect ratio $l / h$ of the individual plates.

Having ascertained the critical design parameters, to test the validity of our analytical model we fabricated surface-attached cellular structures with aspect ratios $l / h$ that correspond to different regimes in the stability diagram, as indicated by the circular markers in Figure 1(b). We started with macro-scale honeycomb structures $(l=5 \mathrm{~mm})$ comprising few unit cells and made from silicone rubber (see Experimental). When swelled, the structures yielded either chiral, achiral or mixed patterns (Figure 2(a) and Supplementary Figure S3), exactly as predicted by our analysis (Figure 1(b)). We then fabricated samples comprising a larger number of unit cells with geometry corresponding to both the achiral $(l / h=2, t / h=0.37$ and $l=5$ $\mathrm{mm})$ and chiral $(l / h=3.17, t / h=0.40$ and $l=5 \mathrm{~mm})$ regime in the stability diagram. Furthermore, since the analytical model is not limited to a specific cellular geometry, we also fabricated macroscale $(l=5 \mathrm{~mm})$ square lattices comprising plates with the same aspect ratio (Figure $2 \mathrm{~b}$ ). Again all the samples buckled as predicted by the analytical model (Figure 2c,d-left/center), showing that the design principles can be applied to different geometries.

Because the pattern formation exploits a mechanical instability that is scaleindependent (where the continuum assumption holds), we extended the principle to microscale honeycombs $(l=20 \mu \mathrm{m})$ made from epoxy resin (see Supplementary Methods). The structures buckled upon immersion in $N$-methyl-2-pyrrolidone, and the stability diagram again correctly predicted the emergence of either chiral or achiral patterns (Figures 2(c,d)-right). Subsequently evaporating the solvent led to reconversion of the buckled structures back into 


\section{Submitted to

the original configurations, demonstrating that the controlled pattern formation is fully reversible.

Although all the experimental results show an excellent agreement with the analytical predictions, we observed a racemic mixture of chiral domains displaying both right- and lefthandedness evolved from several nucleation events. At the boundaries of these domains (shown as yellow lines in Figure 2), the plates buckled either in the $1^{\text {st }}$ or $3^{\text {rd }}$ mode. Avoiding such racemic mixtures and making defect-free patterns with a single-handedness thus requires the prevention of multiple nucleation sites. We therefore gradually wetted the samples from a single location. This approach induced a unique nucleation event that propagated over the entire sample to yield uniform buckling patterns (Figure 3(a)). Remarkably, we observed that during the spreading of the pattern some plates initially buckled either in the wrong direction or with an unpredicted mode (Figure 3(b)). These defects were, however, repaired upon propagation of the swelling front through the defect site when the majority of the plates connected to the defect buckled correctly. The interconnected cellular architecture is therefore crucial to conserve the propagation of a single handedness over the entire substrate (Figure 3(d)) by allowing a self-repair mechanism through connected plates.

While instabilities can be induced by a number of stimuli, including thermal, electrical, and mechanical loadings (Supplementary Figure S4), the convenience, versatility and, most importantly, the reversibility and directionality of the reconfiguration process that arises from swelling-induced buckling enables a unique mechanism where chirality can be chosen, reversibly written and amplified as described below.

Repeated swelling-unswelling revealed that the same buckling pattern reoccurred every time. The structure thus possesses memory of deformation, likely embedded in a previous reorganization of the polymeric network, which can steer the chiral outcome in a 


\section{Submitted to

subsequent symmetry breaking event. This memory effect can be used to select the handedness of the chiral patterns at will. Since statistical analyses showed equal probability of left-handed and right-handed patterns in rapidly swollen structures (Figure 4(a,b)), one can map the domains of different handedness and choose a region of desired handedness as a seed. After unswelling the structure back into the initial pattern, we can initiate the slow, directional swelling of the structure starting from one of the mapped domains (Figure 4(c)). Remarkably, when the progressing pattern reached the previously identified domain boundaries, the handedness of the spreading pattern was still preserved, thereby overwriting the memory of these domains to yield patterns of uniform handedness by the repair mechanism discussed above. When the structure subsequently was unbuckled by drying and rapidly swollen again, we recovered the single-chirality pattern, confirming that the memory of initially racemic domains was indeed overwritten. This chiral memory is also durable: even after four months the memory is still preserved. Information on the handedness can thus be stored, read out, and overwritten providing a versatile programmable mechanism.

Guided by an analytical model and numerical calculations, here we show a proof of principle of buckling-induced local symmetry breaking and subsequent spreading of a chiral pattern to yield globally uniform configurations of single handedness in supported cellular structures. We experimentally demonstrate that this strategy offers a unique range of advantages: (i) it can be applied to structures with various length scales (where the continuum hypothesis holds); (ii) the reconfiguration occurs upon application of different stimuli and using different materials, so that it can be used to dynamically alter surface properties and morphology; (iii) the transformation can be made fully reversible; and (iv) most importantly, it can be controlled to yield either uniform achiral or chiral configurations with user-defined handedness. 


\section{Submitted to

In contrast to molecular symmetry-breaking reactions and crystallization systems, both the nucleation and propagation of chirality can be visualized directly to unravel the underlying mechanisms and fully control the pattern formation. This process not only provides a remarkable example of spontaneous symmetry breaking, but also outlines a general strategy in which a judicious choice of dimensions, materials, stimuli and architectonic designs provides a foundation for a wide range of multidisciplinary basic and applied studies. For example, our results could be used to design templates to facilitate fabrication of free-standing chiral structures. Moreover, in structures with sizes comparable to the wavelength of the light, our approach could lead to the design of novel thin film polarization converters, waveguides and circular dichroism spectroscopy substrates.

\section{Acknowledgements}

This work was supported by Army Research Office MURI award W911NF-09-1-0476 and the Materials Research Science and Engineering Center under NSF Award No. DMR0820484. KB acknowledges support of the Wyss institute through the Seed Grant Program and NSF-CMMI-1149456-CAREER. We acknowledge the use of the facilities at the Harvard Center for Nanoscale Systems supported by NSF Award No. ECS-0335765 and MIT Microsystems Technology Laboratories. WLN acknowledges the Netherlands Organization for Scientific Research for financial support. We thank Dr. James Weaver for help with macroscale structure preparation, Tom de Geus and Bas Overvelde for discussions, and Dr. Jayson Paulose and Dr. Andrej Kosmrlj for helpful comments. (Supporting Information is available online from Wiley InterScience or from the author).

Received: ((will be filled in by the editorial staff))

Revised: ((will be filled in by the editorial staff)) Published online: ((will be filled in by the editorial staff))

\section{References}

[1] S. Zhang, Y. S. Park, J. S. Li, X. C. Lu, W. L. Zhang, X. Zhang, Phys. Rev. Lett. 2009, $102,023901$.

[2] S. Vignolini, N. A. Yufa, P. S. Cunha, S. Guldin, I. Rushkin, M. Stefik, K. Hur, U.

Wiesner, J. J. Baumberg, U. Steiner, Adv. Mater. 2012, 24, OP23.

[3] C. M. Soukoulis, M. Wegener, Nature Photon. 2011, 5, 523. 
Submitted to

\section{ADVANCED}

[4] E. Hendry, T. Carpy, J. Johnston, M. Popland, R. V. Mikhaylovskiy, A. J. Lapthorn, S.

M. Kelly, L. D. Barron, N. Gadegaard, M. Kadodwala, Nature Nanotech. 2010, 5, 783.

[5] J. Jaques, A. Collet, S. H. Wilen, Enatiomers, Racemates and Resolutions, Krieger Publishing Company, Melbourne 1994.

[6] P. Bettini, A. Airoldi, G. Sala, L. Di Landro, M. Ruzzene, A. Spadoni, Compos. Part B-Eng 2010, 41, 133.

[7] M. M. Green, N. C. Peterson, T. Sato, A. Teramoto, R. Cook, S. Lifson, Science 1995, $268,1860$.

[8] S. Bordács, I. Kézsmárki, D. Szaller, L. Demkó, N. Kida, H. Murakawa, Y. Onose, R.

Shimano, T. Rõõm, U. Nagel, S. Miyahara, N. Furukawa, Y. Tokura, Nature Phys. 2012, 8, 734.

[9] K. Soai, T. Shibata, H. Morioka, K. Choji, Nature 1995, 378, 767.

[10] D. K. Kondepudi, R. J. Kaufman, N. Singh, Science 1990, 250, 975.

[11] C. Viedma, Phys. Rev. Lett. 2005, 94, 065504.

[12] W. L. Noorduin, T. Izumi, A. Millemaggi, M. Leeman, H. Meekes, W. J. P. Van Enckevort, R. M. Kellogg, B. Kaptein, E. Vlieg, D. G. Blackmond, J. Am. Chem. Soc. 2008, $130,1158$.

[13] F. C. Frank, Biochim. Biophys. Acta 1953, 11, 459.

[14] B. Pokroy, S. H. Kang, L. Mahadevan, J. Aizenberg, Science 2009, 323, 237.

[15] S. H. Kang, N. Wu, A. Grinthal, J. Aizenberg, Phys. Rev. Lett. 2011, 107, 177802.

[16] A. Grinthal, S. H. Kang, A. K. Epstein, M. Aizenberg, M. Khan, J. Aizenberg, Nano Today 2012, 7, 35 .

[17] S. Singamaneni, M. E. McConney, V. V. Tsukruk, ACS Nano 2010, 4, 2327.

[18] S. Singamaneni, M. E. McConney, V. V. Tsukruk, Adv. Mater. 2010, 22, 1263.

[19] Y. Forterre, J. M. Skotheim, J. Dumais, L. Mahadevan, Nature 2005, 433, 421.

[20] P. D. Shipman, A. C. Newell, Phys. Rev. Lett. 2004, 92, 168102. 
[21] E. P. Chan, A. J. Crosby, Adv. Mater. 2006, 18, 3238.

[22] Y. Zhang, E. A. Matsumoto, A. Peter, P. C. Lin, R. D. Kamien, S. Yang, Nano Lett. 2008, $8,1192$.

[23] J. H. Jang, C. Y. Koh, K. Bertoldi, M. C. Boyce, E. L. Thomas, Nano Lett. 2009, 9, 2113.

[24] S. Singamaneni, V. V. Tsukruk, Soft Matter 2010, 6, 5681.

[25] Y. Rahmawan, M. W. Moon, K. S. Kim, K. R. Lee, K. Y. Suh, Langmuir 2010, 26, 484.

[26] J. B. Kim, P. Kim, N. C. Pegard, S. J. Oh, C. R. Kagan, J. W. Fleischer, H. A. Stone, Y. L. Loo, Nature Photon. 2012, 6, 327.

[27] K. Bertoldi, P. M. Reis, S. Willshaw, T. Mullin, Adv. Mater. 2010, 22, 361.

[28] J. Shim, C. Perdigou, E. R. Chen, K. Bertoldi, P. M. Reis, Proc. Natl. Acad. Sci. USA 2012, 109, 5978.

[29] V. R. Tirumala, C. M. Stafford, L. E. Ocola, J. F. Douglas, L. Mahadevan, Nano Lett. 2012, 12, 1516.

[30] L. J. Gibson, M. F. Ashby, Cellular Solids: Structure and Properties, Cambridge University Press, Cambridge, U.K. 1988.

[31] D. Prall, R. S. Lakes, Int. J. Mech. Sci. 1997, 39, 305.

[32] A. Spadoni, M. Ruzzene, F. Scarpa, Phys. Status Solidi B 2005, 242, 695.

[33] L. Baggetto, D. Danilov, P. H. L. Notten, Adv. Mater. 2011, 23, 1563.

[34] S. Timoshenko, J. M. Gere, Theory of elastic stability, McGraw-Hill, New York 1961.

[35] B. Pokroy, A. K. Epstein, M. C. M. Persson-Gulda, J. Aizenberg, Adv. Mater. 2009, $21,463$. 


\section{Figures and Captions}
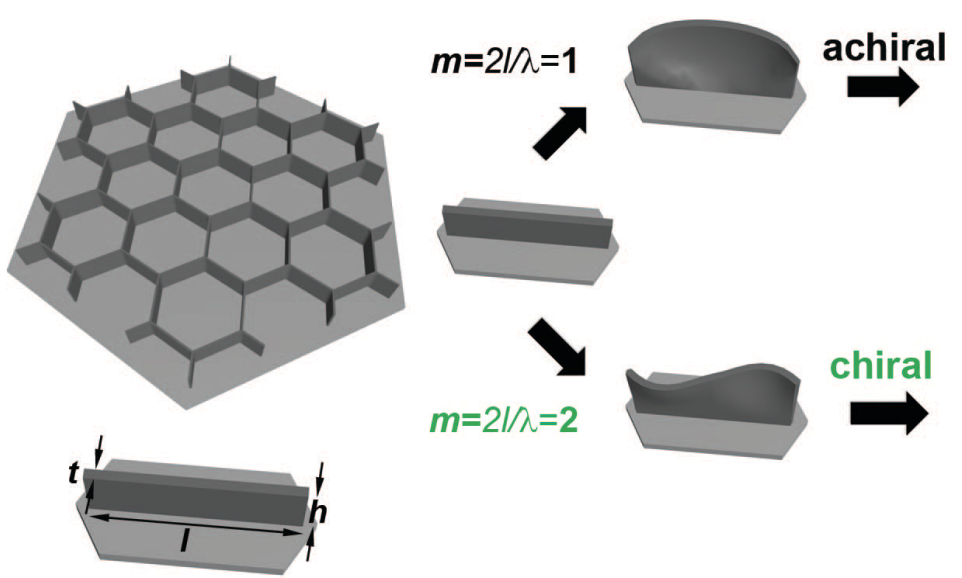

Reconstruction
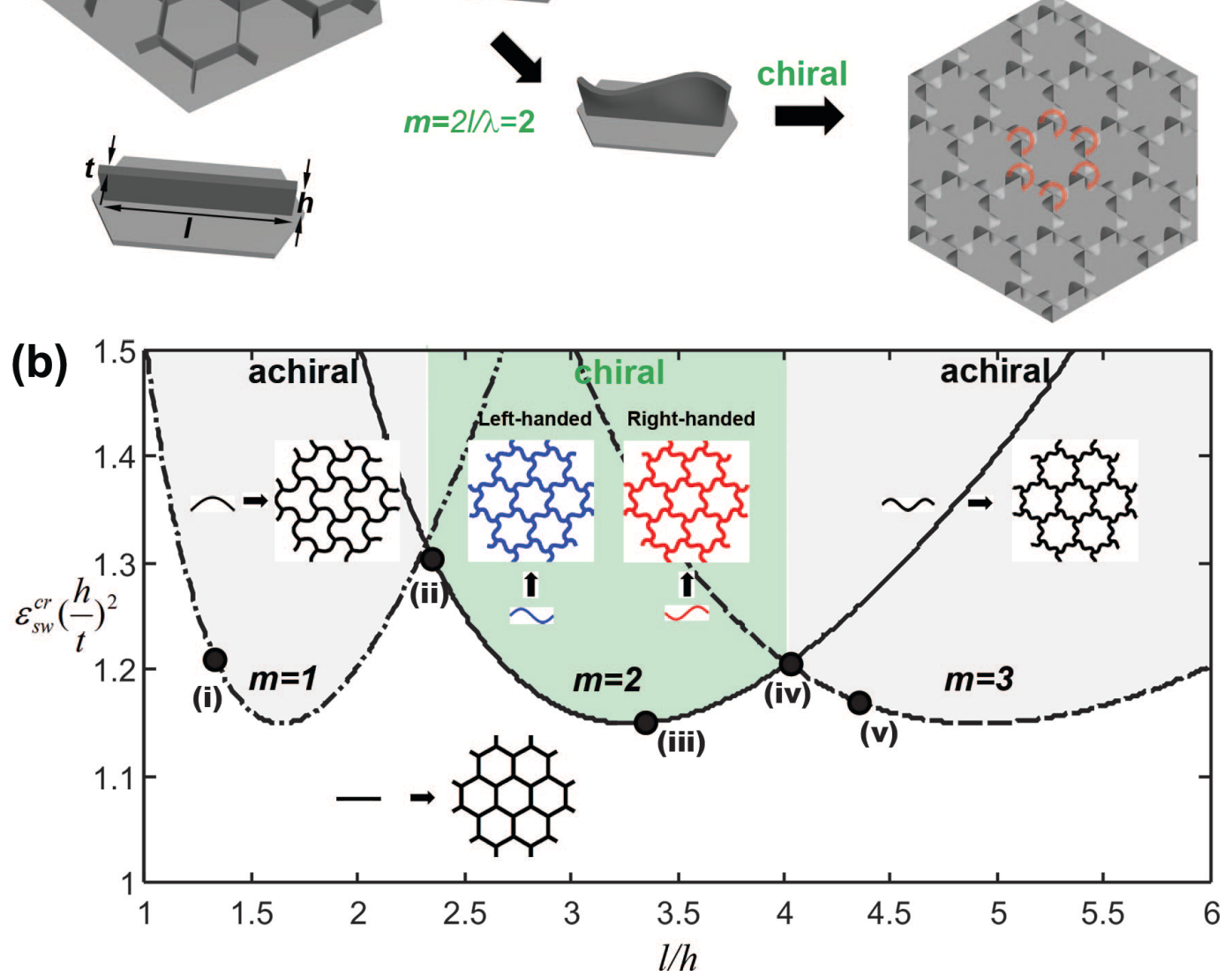

Figure 1. Design of structures. (a) Schematic approach to the design of supported cellular architectures with buckling-induced chiral or achiral reconfigurations. First, the cellular structure is deconstructed into individual supported plates and the wavelength $\lambda$ of their buckling pattern is calculated. Then, the buckled pattern of the structure is reconstructed by connecting individual buckled plates. The color-coded arrows indicate the handedness of the vertices. (b) Results of the buckling analysis for a single supported thin, elastic plate are plotted in a stability diagram. Upon reaching a critical swelling strain $\varepsilon_{s w}^{c r}$, buckling modes of 
both half and full sinusoids can be achieved by adjusting $l / h$ (the aspect ratio of the plate), resulting in respectively achiral and chiral patterns upon reconstruction of the cellular architecture. The circular black markers and corresponding Roman numbers indicate the aspect ratio $l / h$ of macroscale honeycomb lattices $(l=5 \mathrm{~mm}$ and $t / l=0.18$, see Figure $2(\mathrm{a}))$ that were fabricated and tested to verify the validity of the analytical model. 


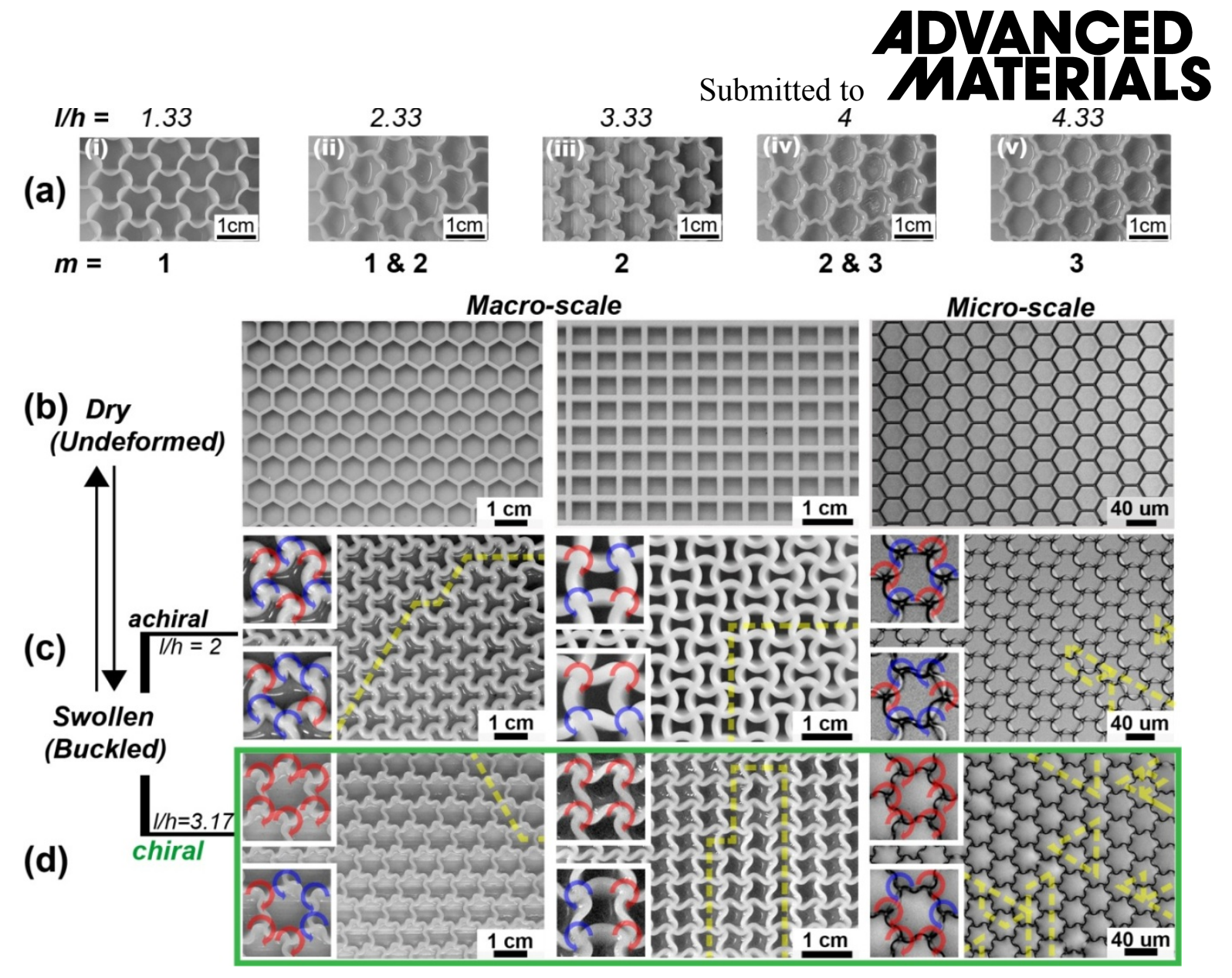

Figure 2. Verification of the stability diagram. (a) Buckling patterns of macroscale honeycombs with different aspect ratio $l / h(t / l=0.18$ and $l=5 \mathrm{~mm}$ for all samples $)$. The Roman numbers indicate the corresponding marker in the stability diagram (Figure 1(b)). The observed buckling mode $(m)$ is shown below each image. Note that a mixture of modes is found for geometries lying on the boundary of adjacent regimes. (b)-(d) Buckling-induced reversible pattern formation in a supported macro-scale honeycomb lattice (left), macro-scale square lattice (center) and micro-scale honeycomb lattice (right) upon rapid swelling. (b) Optical images of original, undeformed structures. (c) For $l / h=2, t / h=0.37$, buckling induces an achiral pattern. (d) For $l / h=3.17, t / h=0.40$, a chiral pattern is observed. These buckling patterns are in agreement with the analytical predictions, but multiple domains are observed, whose boundaries are highlighted by the yellow dashed lines. The insets show zoomed-in images of the buckled patterns within the domains (top) and at the domain boundaries (bottom). The color-coded arrows indicate the handedness of the vertices. 
(a)
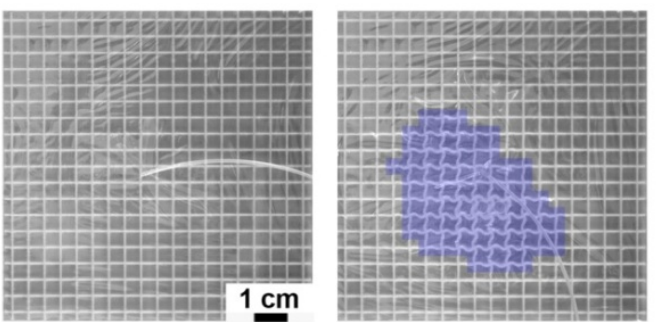

Submitted to

(b)
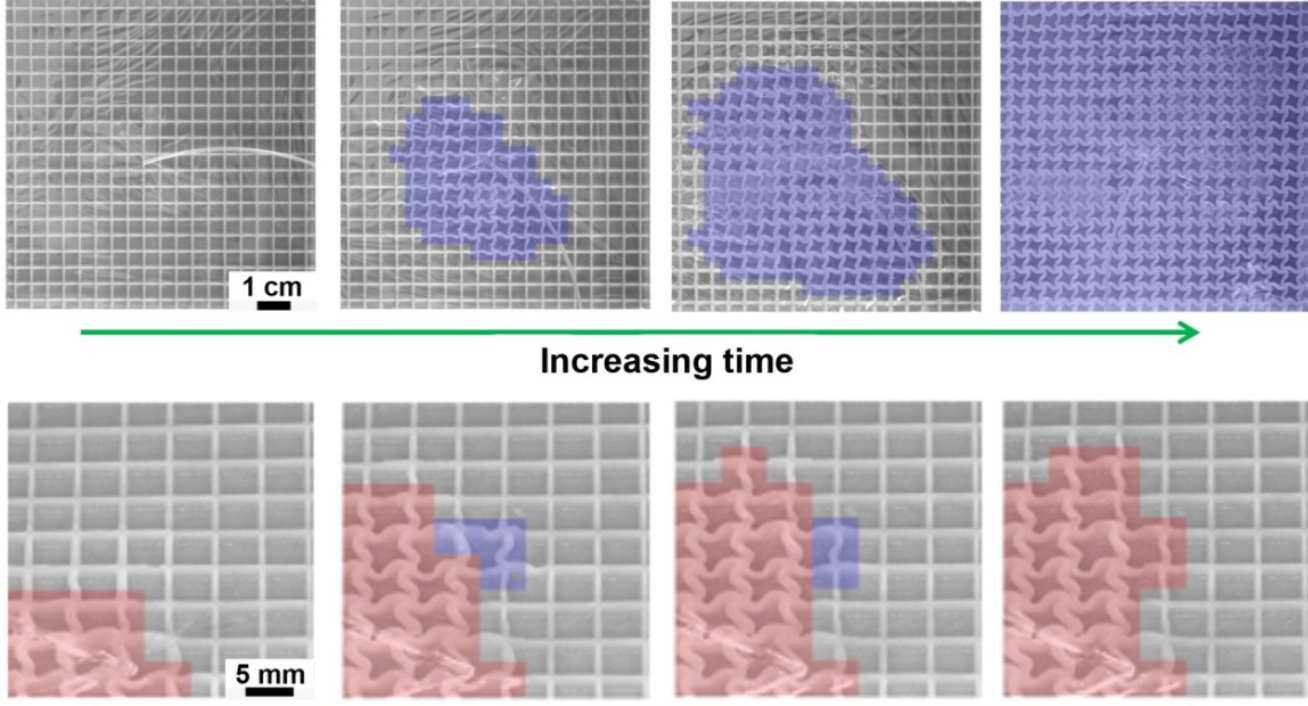

Increasing time

Increasing time

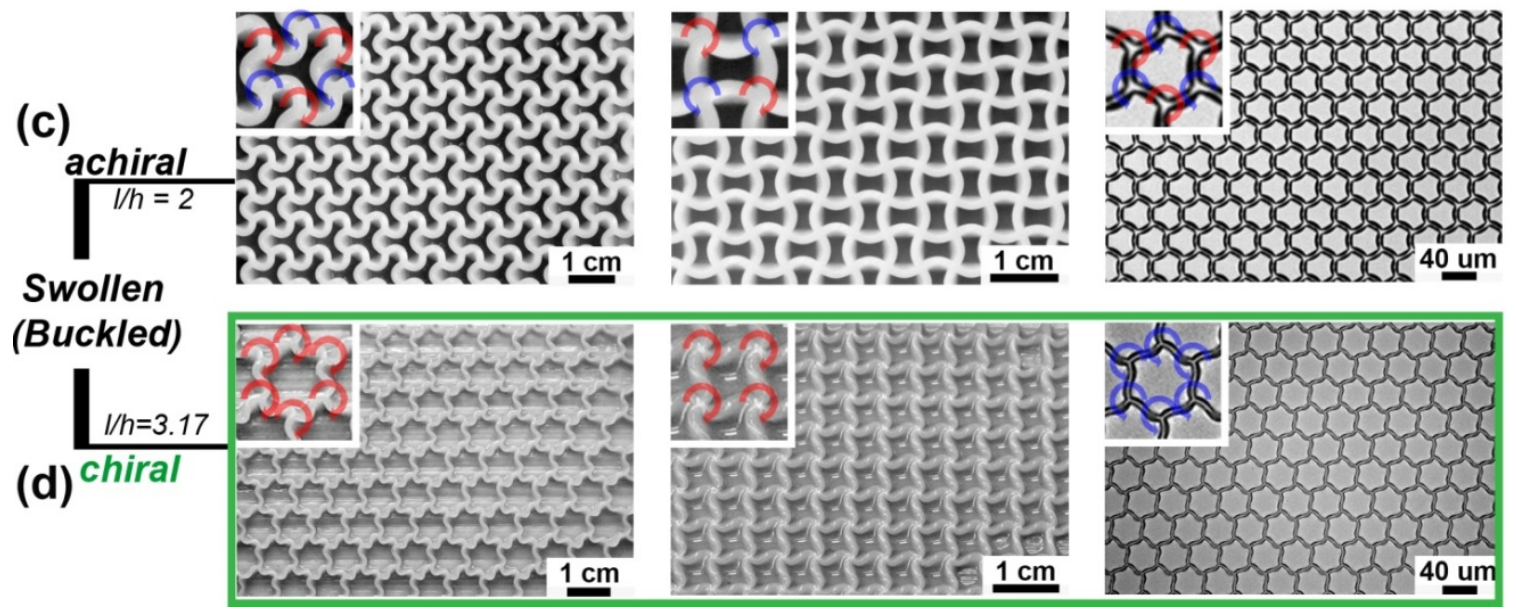

Figure 3. Uniform pattern formation by controlling nucleation. (a) Time-lapse series showing a single nucleation event and subsequent slow spreading of a buckling-induced chiral pattern by gradually wetting from a single location. (b) Time-lapse series of the self-repairing process. The initial defect in the form of few clockwise vertices (blue) is overwhelmed by the surrounding counterclockwise vertices (red), thus amplifying the chiral patterns of the nucleus. (c)-(d) The combination of the unique nucleation event and amplification mechanism results in either uniform achiral $(l / h=2, t / h=0.37)$ (c) or chiral $(l / h=3.17, t / h=0.40)$ (d) patterns. Zoomed-in images of the buckled patterns are shown in the insets; the color-coded arrows indicate the handedness of the vertices. 
(a)

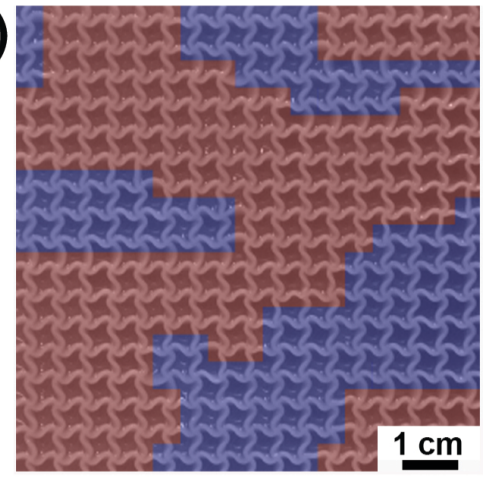

Submitted to

(b)

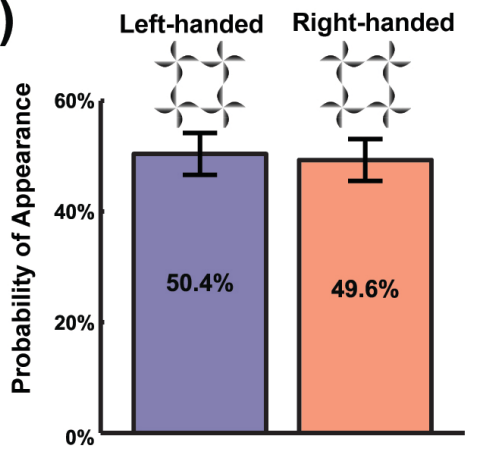

(c) Original structure
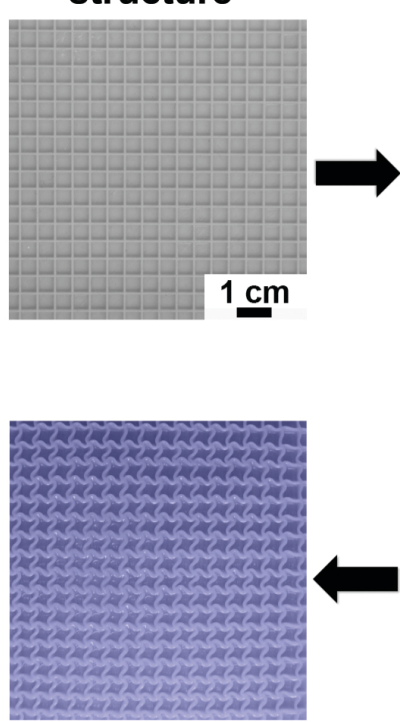

After swelling
After swelling
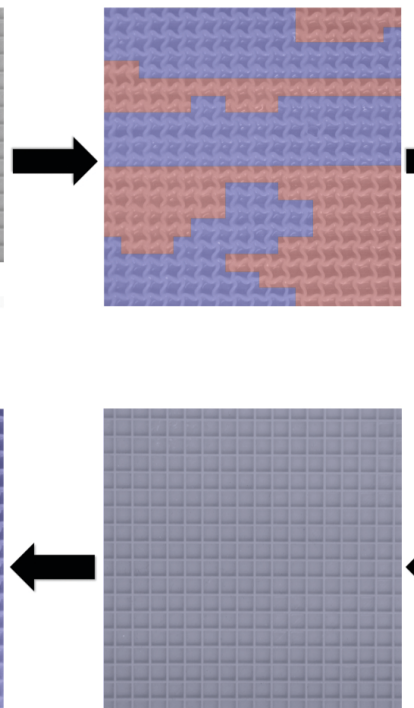

After unswelling

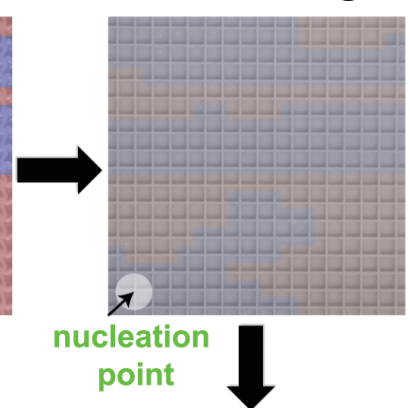

nucleation point

After unswelling
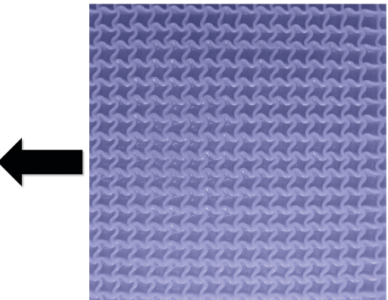

After chiral amplification

Figure 4. Memory effect allowing readout, selection and overwriting of the handedness of the chiral pattern. (a) An example of a rapidly swollen macro-scale square lattice structures, showing a racemic mixture of left- and right-handed domains. (b) Statistical probabilities of the vertices rotate into right- and left-handed configurations from nine independent experiments using different samples. The error bar indicates the standard deviation of the respective probability. (c) These chiral domains can be selected and amplified. For this, we first map the chiral domains upon rapid swelling. Subsequently the structure is reconverted into the unbuckled configuration, where the handedness map is given in faded red and blue. Buckling is then initiated at a location selected from the chirality map to induce a uniform pattern of the desired handedness. If the structure is subsequently unbuckled and rapidly 
Submitted to

\section{ADANACEPS
MATERRALIS}

swollen, the uniform pattern is recovered, demonstrating that the chiral memory of the original racemic pattern is overwritten.

\section{Experimental}

Fabrication of samples: Macroscale $(l=5 \mathrm{~mm})$ surface-attached hexagonal and square cellular structures were fabricated by first making negative molds using a 3D printer (Connex 500 available from Objet, Ltd.) that subsequently were used to make positive structures from a silicone rubber (Elite Double 32 available from Zhermack). The structures were attached to rigid back plates. Two microscale surface-attached hexagonal structures $(l=20 \mu \mathrm{m})$ were fabricated for this study. The aspect ratios of the macroscale and microscale plates are $(l / h=2$, $t / h=0.37)$ and $(l / h=3.17, t / h=0.40)$ for achiral and chiral structures, respectively. The microscale structures were first fabricated in $<100>$ Si wafers. Replicas of the silicon masters were fabricated using soft lithography-based methods ${ }^{[35]}$ with commercially available UVcurable epoxy (UVO-114 from Epoxy Technology, Billerica, MA).

Buckling-induced pattern formation: Buckling was induced by swelling, using hexane and N-methyl-2-pyrrolidone (NMP) for silicon rubber and epoxy structures, respectively. The resulting patterns were recorded using a Nikon D90 digital SLR camera for the macroscale samples and a Leica DMRX microscope connected to a QImaging Evolution VF cooled color CCD camera for the microscale samples. 


\section{The table of contents entry}

\section{Submitted to

Keyword: buckling, chirality, handedness, reversibility, cellular structures

Authors: Sung Hoon Kang ${ }^{\dagger}$, Sicong Shan ${ }^{\dagger}$, Wim L. Noorduin ${ }^{\dagger}$, Mughees Khan, Joanna Aizenberg*, and Katia Bertoldi* (†equal contribution, *corresponding author)

Title: Buckling-Induced Reversible Symmetry Breaking and Amplification of Chirality Using Supported Cellular Structures

ToC figure:

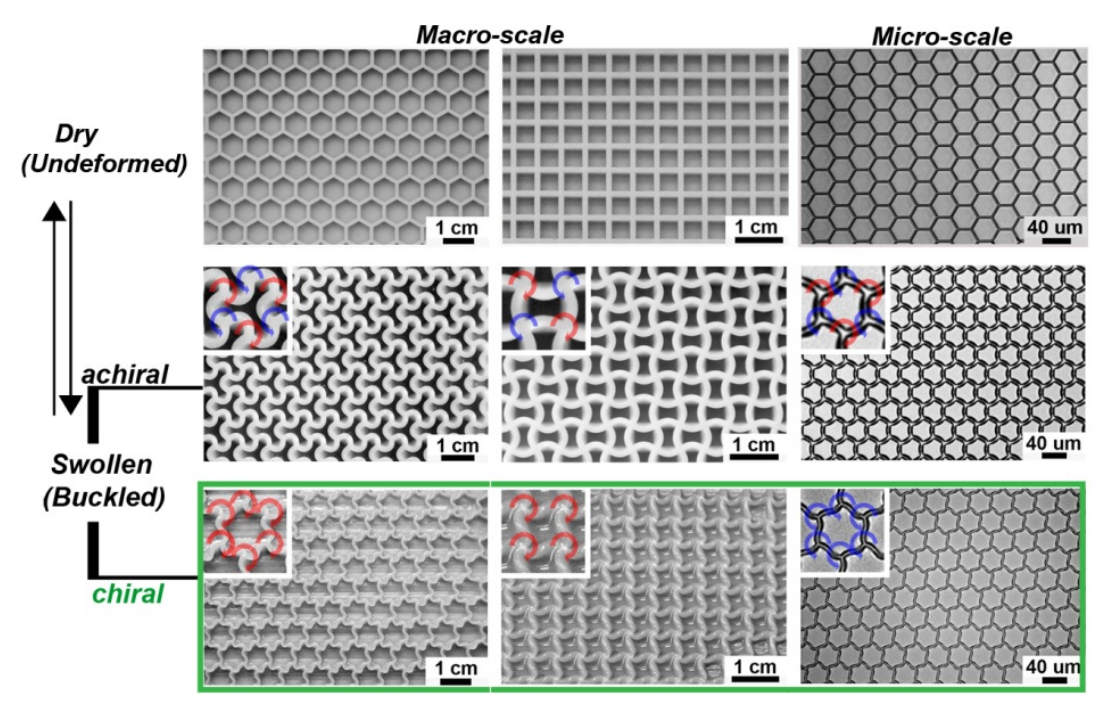

Buckling-induced reversible pattern formation in a supported macro-scale honeycomb lattice (left), macro-scale square lattice (center) and micro-scale honeycomb lattice (right) upon swelling. The combination of the unique nucleation event and amplification mechanism results in either uniform achiral or chiral patterns. Zoomed-in images of the buckled patterns are shown in the insets; the color-coded arrows indicate the handedness of the vertices. 


\section{Supplementary Methods}

\section{Theoretical model for buckling of a supported elastic plate.}

To gain more insight into the response of surface attached cellular structures, we start by investigating buckling of the individual cell edges consisting of rectangular plates of length $l$, height $h$ and thickness $t$ (see Figure 1(a)) subjected to isotropic swelling.

Foppl-von Karman equations. For the sake of simplicity, we focus on thin plates, so that shear deformations through the thickness of the plate can be neglected, and consider a deformation that takes a point on the center-surface with coordinates $(x, y, 0)$ to its deformed state $\left(x+u_{x}(x, y), y+u_{y}(x, y), w(x, y)\right)$. Assuming that the thin plate may be described as a linear elastic material with Young's modulus $E$, Poisson's ratio $v$, and bending stiffness $D=E$ $t^{3} /\left[12\left(1-v^{2}\right)\right]$, then balance of forces in the plane and out of the plane directions leads to the Foppl-von Karman equations [1-2]

$$
\sigma_{i j, j}=0, \quad \mathrm{D}\left(\mathrm{w}_{, \mathrm{xxxx}}+\mathrm{w}_{, \mathrm{yyyy}}+2 \mathrm{w}_{, \mathrm{xxyy}}\right)-\mathrm{tw}_{, \mathrm{ij}} \sigma_{, i j}=0, \quad i, j=x, y
$$

where $A_{, x}=\partial A / \partial x$. Assuming isotropic swelling, the in-plane stresses are given by

$$
\begin{gathered}
\sigma_{i j}=\frac{E}{1+\nu}\left[\varepsilon_{i j}+\frac{\nu}{1-\nu}\left(\varepsilon_{x x}+\varepsilon_{y y}\right) \delta_{i j}\right]- \\
\frac{E}{1-v} \varepsilon_{s w} \delta_{i j}
\end{gathered}
$$

where $\delta_{i j}$ is the Kronecker delta, $\varepsilon_{i j}=\frac{1}{2}\left(u_{i, j}+u_{j, i}\right)+\frac{1}{2} w_{, i} w_{, j}$ is the in-plane strain tensor and $\varepsilon_{s w}$ denotes the strain caused by swelling of the material. 
Boundary conditions. In a $2 \mathrm{D}$ cellular structure attached to a substrate that is very stiff and swells by a negligible amount, clamped conditions can be assumed for each individual plate at $y=0$,

$$
u_{x}(x, 0)=u_{y}(x, 0)=w(x, 0)=w_{, y}(x, 0)=0
$$

while the condition that the boundary at $y=h$ is free of torques and forces implies that

$$
\begin{gathered}
\sigma_{y y}(x, h)=\sigma_{x y}(x, h)=0, \\
w_{, y y}(x, h)+v_{w_{, x x}}(x, h)=w_{, y y y}(x, h)+(2-v) w_{, x x y}(x, h)=0 .
\end{gathered}
$$

Finally, in a lattice where the junctions cannot move but are free to rotate for each plate the boundary conditions at $x=0, l \mathrm{read}$

$$
u_{x}(x, y)=w(x, y)=0, w_{, x x}(x, y)+v_{w_{, y y}}(x, h)=0, \text { at } x=0, l
$$

Buckling. We start by noting that Eqns. (1)-(5) are identically satisfied when

$$
u_{x}(x, y)=w(x, y)=0, u_{y}(x, y)=\varepsilon_{s w}(1+v) y
$$

resulting in uniform strains and stresses and zero deflection. However, this solution is not always stable and above a critical value of swelling strain, $\varepsilon_{s w}^{c r}$, non-planar solutions appear. To test the stability of the solution Eq. (6), we introduce incremental deformations as follows

$$
\tilde{u}_{i}(x, y)=u_{i}(x, y)+\varepsilon u_{i}^{(1)}(x, y), \widetilde{w}(x, y)=w(x, y)+\varepsilon w^{(1)}(x, y)
$$

where $\varepsilon$ is a small parameter that characterizes the size of the perturbation superimposed on the finite deformation. Introducing Eq. (7) in Eq. (1) and retaining only the first order terms in $\varepsilon$, a single non-trivial incremental equilibrium equation is obtained 


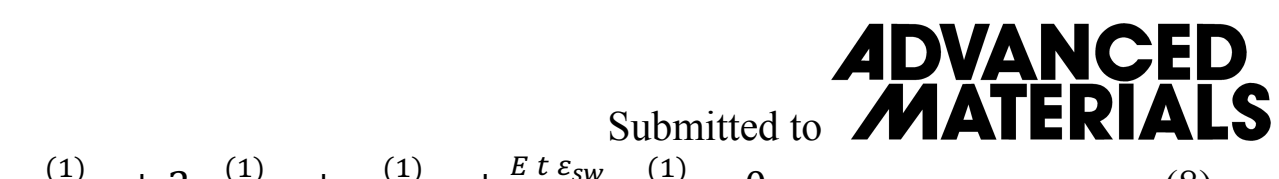$$
w_{, x x x x}^{(1)}+2 w_{, x x y y}^{(1)}+w_{, y y y y}^{(1)}+\frac{E t \varepsilon_{s w}}{D} w_{, x x}^{(1)}=0
$$

Assuming the out-of-plane deflection is of the form $w^{(1)}(x, y)=f(y) \sin \frac{m \pi x}{l}(m$ being an integer) on substituting it into Eq. (8) and using the boundary conditions (3)-(5), an ordinary differential equation for $f(y)$ is obtained

$$
f_{, y y y y}-\frac{2 m^{2} \pi^{2}}{l^{2}} f_{, y y}+\left(\frac{m^{4} \pi^{4}}{l^{4}}-\frac{E t \varepsilon_{s w}}{D} \frac{m^{2} \pi^{2}}{l^{2}}\right) f=0
$$

The general solution of a fourth order differential equation like Eq. (9) is given as,

$$
f(y)=C_{1} e^{-\alpha y}+C_{2} e^{\alpha y}+C_{3} \cos (\beta y)+C_{4} \sin (\beta y)
$$

Where $\alpha$ and $\beta$ are as following,

$$
\alpha=\sqrt{\frac{m^{2} \pi^{2}}{l^{2}}+\sqrt{\frac{m^{2} \pi^{2} E t \varepsilon_{s w}}{D l^{2}}}}, \beta=\sqrt{-\frac{m^{2} \pi^{2}}{l^{2}}+\sqrt{\frac{m^{2} \pi^{2} E t \varepsilon_{s w}}{D l^{2}}}} .
$$

The coefficients $\mathrm{C}_{1}, \mathrm{C}_{2}, \mathrm{C}_{3}$ and $\mathrm{C}_{4}$ in Eq. (10) are determined by imposing the boundary conditions (3)-(5). More specifically, the boundary conditions (3) are satisfied if

$$
C_{1}=\frac{C_{4} \beta-C_{3} \alpha}{2 \alpha}, \quad C_{2}=-\frac{C_{3} \alpha+C_{4} \beta}{2 \alpha}
$$

and the function $f(y)$ can be represented in the form

$$
f(y)=C_{3}[\cos (y \beta)-\cosh (y \alpha)]+C_{4}\left[\sin (y \beta)-\frac{\beta}{\alpha} \sinh (y \alpha)\right]
$$

Substituting the above expression in Eq. (4), we get two linear homogeneous equations in terms of $C_{3}$ and $C_{4}$. The critical value of swelling strain, $\varepsilon_{s w}^{c r}$, is determined by equating to zero the determinant of these equations. 


\section{Submitted to

We solve this boundary value problem numerically and study the normalized critical strain $\frac{h^{2} \varepsilon_{S W}^{c r}}{t^{2}}$ and corresponding buckling modes as a function of the plate aspect ratio $l / h$, as shown in Figure 1(b). It is interesting to observe that higher buckling modes (i.e. $m>1$ ) can be achieved for increasing values of $l / h$. By contrast, we notice that for a plate with free edges both at $\mathrm{y}=0$ and $\mathrm{y}=h$ only modes with half wavelength (i.e. $m=1$ ) are possible.

\section{Numerical simulations of buckling for supported cellular structures}

Since our analytical model is based on the theory for thin elastic plates, to fully account for the effect of thickness, we further investigated the buckling of surface-attached lattice structures using non-linear finite element (FE) analyses. The FE calculations were conducted within the nonlinear code ABAQUS/Standard, version 6.8-2. In the numerical analyses, buckling of cellular structures of infinite planar extent perfectly bonded to a rigid substrate was investigated and for that sake of computational efficiency, the analyses were conducted on representative volume elements (RVEs) (see Figure S1).

The instability of the structures was investigated conducting a Bloch wave analysis [5-6], a staple of physics long used to examine electronic, photonic and phononic band structures in periodic lattices. Although instabilities may alter the initial periodicity of the solid, they can be still detected investigating the response of just one unit cell of the material introducing boundary conditions provided by Bloch theory. While a real natural frequency corresponds to a propagating wave, a complex natural frequency identifies a perturbation exponentially growing with time. Therefore, the transition between a stable and an unstable configuration is detected when the frequency vanishes and the new periodicity after instability is provided by the corresponding Bloch wave vector. Here, the finite-element method was used to perform the Bloch wave analysis [5]. 
FE models were constructed using 20-nodes, quadratic elements (ABAQUS element type C3D20). The accuracy of each mesh was ascertained through a mesh refinement study. In the analysis the bottom surface of the structures was considered to be perfectly bonded to a rigid substrate, while the top surface was free. Moreover, a series of constraint equations were applied to the lateral surfaces of the RVE providing Floquet quasi-periodic boundary conditions. The material was modeled as elastic nearly incompressible Neo-Hookean material and swelling was used to induce buckling.

The results for the four structures in the Figure S2 showed the same buckling mode predicted by the analytical model based on Foppl-von Karman equations. More specifically, for $l / h=2$ and $t / h=0.37$ buckling induces the formation of an achiral pattern with reduced symmetry for both the hexagonal and square architecture. Differently, a chiral pattern is induced by mechanical instabilities when $l / h=3.17$ and $t / h=0.40$. Finally, we note that for a square lattice with $l / h=2$ and $t / h=0.37$ at the onset of instability the initial periodicity is broken, leading to an enlarged RVE with $2 \times 2$ original RVEs.

Furthermore, the numerical calculations clearly show that the thickness only marginally affects the buckling modes. In Figure S2(b) we report the critical modes for supported square lattices with $t / h=0.1,0.3$ and 0.5 . These results demonstrate that the buckling mode is not affected by $t / h$. The numerical simulations thus confirm that by simply controlling the aspect ratio $l / h$ of supported-cellular structure in initial honeycomb structure either achiral or chiral configurations can be formed.

\section{Experimental verification of the stability diagram}

Having ascertained the critical design parameters, to verify the validity of our analytical model we fabricated surface-attached cellular structures with aspect ratio $l / h$ that correspond to different domains in the stability diagram. More specifically, we started with macro-scale 
honeycomb structures $(l=5 \mathrm{~mm})$ comprising 19 hexagonal units and made from silicone rubber (see Fabrication section for details). We fabricated 13 samples characterized by $l=5$ $\mathrm{mm}, t=0.9 \mathrm{~mm}$ and $h$ such that $l / h=1,1.33,1.66,2,2.33,2.66,3,3.33,3.66,4,4.33,4.66$ and 5 , as indicated by the markers in Figure S3(a). Immersing the sample in hexane swelled the polymer, and within a few seconds the initially straight cell walls buckled to yield either chiral, achiral or mixed patterns (Figure S3(b)), exactly as predicted by the stability diagram (Figure 1(b)). This set of experimental results clearly confirms the validity of our analytical model.

\section{Fabrication}

Macro-scale structures. Macro-scale $(l=5 \mathrm{~mm})$ surface-attached hexagonal and square cellular structures were fabricated by making negative molds using a 3D printer (Connex 500 available from Objet, Ltd.) and replicating positive structures with a silicone rubber (Elite Double 32 available from Zhermack). Before replication, a releasing agent (Easy Release 200 available from Smooth-On, Inc.) was sprayed on to the molds for easy separation. The structures were attached to rigid back plates.

Micro-scale structures. Two micro-scale different surface-attached hexagonal structures were fabricated for this study, with aspect ratios that corresponded to both achiral $(l / h=2$, $t / h=0.37)$ and chiral $(l / h=3.17, t / h=0.40)$ structures. The honeycomb structures yielding an achiral buckled pattern comprise individual plates with a thickness of $3.7 \mu \mathrm{m}$, a length of 20 $\mu \mathrm{m}$, and a height of $10 \mu \mathrm{m}$. The prochiral structures consist of an array of plates with thickness of $2.5 \mu \mathrm{m}$, a length of $20 \mu \mathrm{m}$, and a height of $6.3 \mu \mathrm{m}$. The different aspect ratio honeycomb structures used for this study were first fabricated in $<100>$ Si wafers. The wafers were vapor primed with Hexamethyldisilazane (HMDS) to improve photo resist adhesion. SPR700 was used as a resist. Nikon $5 \times$ i-line stepper was used to expose the coated wafers 
Submitted to

followed by post exposure bake and resist development using Microposit MF CD26.

Descumming was performed prior to Bosch advanced silicon etch [3] in an STS deep reactive ion etching (DRIE) tool. $\mathrm{C}_{4} \mathrm{~F}_{8}$ and $\mathrm{SF}_{6}$ chemistry was used for Si etching. Veeco profilometers and cross sectional SEM (Zeiss) was used to characterize and optimize exposure conditions etch rate and etch profile. The $\mathrm{Si}$ wafers were given a final overcoat of $\mathrm{C}_{4} \mathrm{~F}_{8}$ to aid in the replication process. Scalloping on the plates of honeycomb structures is the result of the Bosch etch process [3-4] and is also visible in the polymer replicas. Replicas of the silicon masters were fabricated using soft lithography-based methods with commercially available UV-curable epoxy (UVO-114 from Epoxy Technology, Billerica, MA) as described in a previous paper [7].

\section{Buckling-induced pattern formation through swelling}

Swelling was used to trigger instability in the considered supported cellular structures. Buckling occurred due to compressive stresses arising from the geometric constraint that suppresses swelling near the rigid substrate where the structure is clamped.

Macro-scale structures. Instability of fabricated structures was induced by swelling the structures with hexane. We started by rapidly immersing the samples in hexane. We observed the formation of buckled patterns in excellent agreement with the analytical predictions, but characterized by multiple domains that evolved from different nucleation sites. To make defect-free samples, we carefully controlled nucleation and propagation of the pattern. A plastic tube (Intramedic polyethylene tubing with $\mathrm{ID}=0.38 \mathrm{~mm}, \mathrm{OD}=1.09 \mathrm{~mm}$ available from Becton, Dickinson and Company) was used to gradually wet the samples starting from a single location and the solvent was introduced through a syringe pump (PHD 2000 available from Harvard Apparatus) at a flow rate of $0.1-0.4 \mathrm{~mL} / \mathrm{min}$. The resulting patterns were recorded using Nikon D90 digital SLR camera. 
Micro-scale structures. Buckling was induced by swelling the structures with N-methyl-2pyrrolidone (NMP) (available from Sigma Aldrich). We started by rapidly immersing the structures in the solvent, leading to the formation of multiple domains. To make defect-free samples, we floated them on the solvent so that the structure swelled from one side by wetting. The resulting patterns were recorded by optical microscope (Leica DMRX connected to a QImaging Evolution VF cooled color CCD camera).

\section{Buckling-induced pattern formation through compression}

We demonstrated that the pattern formation can be extended to different stimuli, by inducing buckling through mechanical loading. We performed compression experiment on microscale honeycomb structures made of UV curable epoxy and comprising an array of plates with a thickness of $3.7 \mu \mathrm{m}$, a length of $20 \mu \mathrm{m}$, and a height of $10 \mu \mathrm{m}(l / h=2, t / h=0.37)$. Figure S4 shows the compressed (left) vs. uncompressed (right) areas of a single sample. An exceptionally uniform achiral buckled pattern is observed in the compressed area.

\section{References}

[1] A. Föppl, Vorlesungen über technische Mechanik, B.G. Teubner, Leipzig, Germany 1907.

[2] T. von Kármán, Festigkeitsproblem im Maschinenbau, Vol. IV, Teubner, Stuttgart 1910.

[3] N. Maluf, An Introduction to Microelectromechanical Systems Engineering Artech House, Norwood, MA 2000.

[4] A. A. Ayon, R. Braff, C. C. Lin, H. H. Sawin, M. A. Schmidt, J. Electrochem. Soc. 1999, 146, 339.

[5] K. Bertoldi, M. C. Boyce, Phys. Rev. B 2008, 78, 184107. 
[6] N. Triantafyllidis, M. D. Nestorovic, M. W. Schraad, J. Appl. Mech.-Trans. ASME 2006, 73, 505 .

[7] B. Pokroy, A. K. Epstein, M. C. M. Persson-Gulda, J. Aizenberg, Adv. Mater. 2009, $21,463$.

\section{Supplementary Figures and Captions}
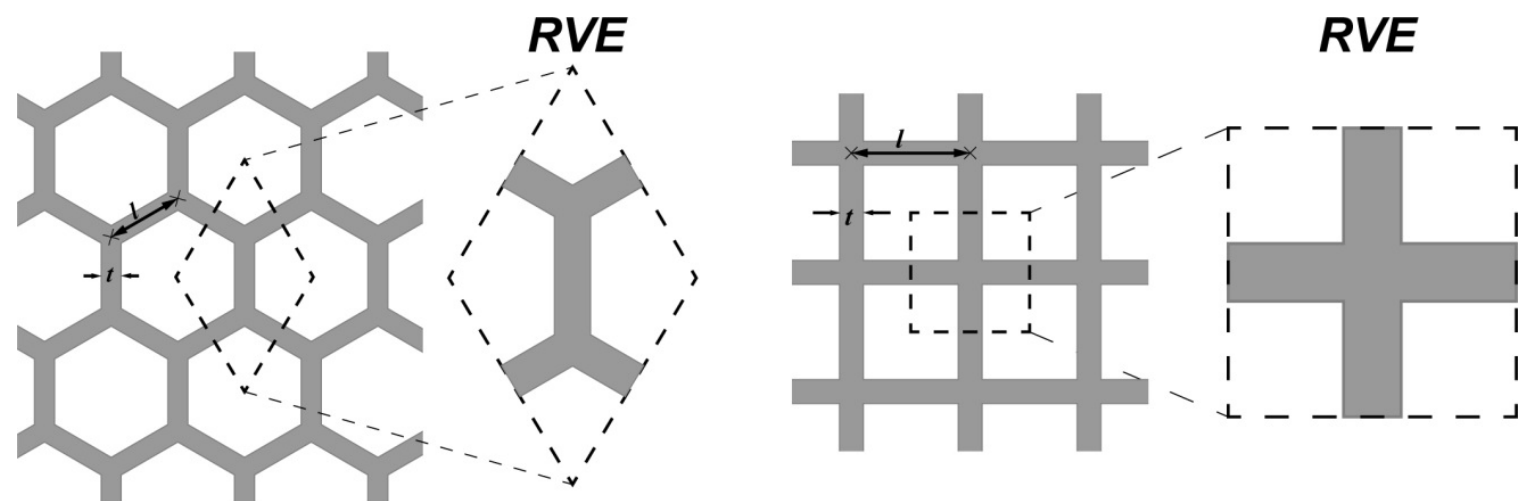

Supplementary Figure S1. Top views of hexagonal and square cellular structures. Notice that $l$ is defined as the center-to-center distance between adjacent vertices. The magnified portions of the structure show the representative volume elements (RVE) of the corresponding architecture. 


\section{ADANCEDS
MAATRRALIS}

(a)

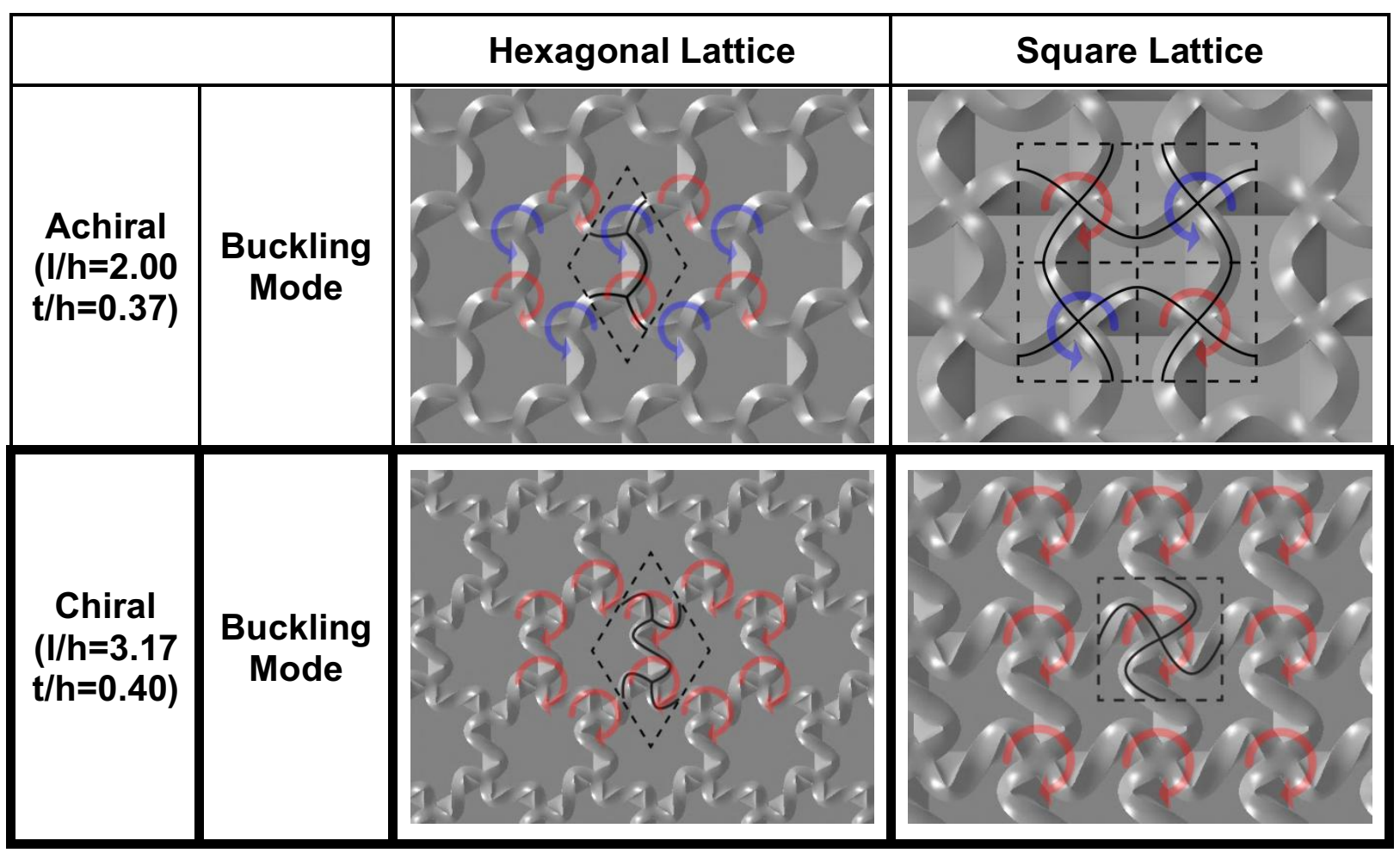

(b)

\begin{tabular}{|c|c|c|c|}
\hline & $\mathrm{t} / \mathrm{h}=0.1$ & $\mathrm{t} / \mathrm{h}=0.3$ & $\mathrm{t} / \mathrm{h}=0.5$ \\
\hline & & & \\
$\begin{array}{c}\text { Achiral } \\
(\mathrm{I} / \mathrm{h}=2.00)\end{array}$ & & & \\
\end{tabular}




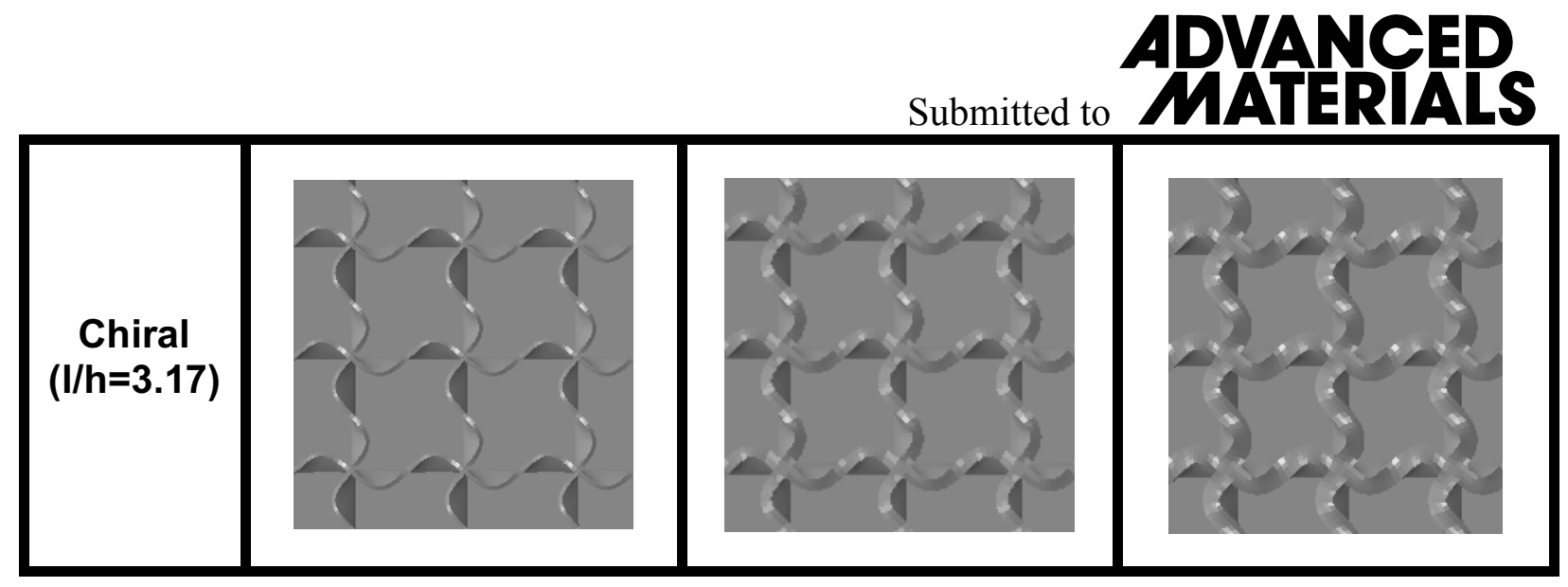

Supplementary Figure S2. (a) Critical buckling modes predicted by numerical analysis. Buckling modes predicted by the Bloch wave analysis for supported hexagonal and square lattices with $(l / h=2, t / h=0.37)$ and $(l / h=3.17, t / h=0.40)$. The handedness of adjacent vertices has been highlight with red (right-handed) or blue (left-handed) arrows. The top views of buckled patterns are outlined by solid curves on the top surface of the structure to facilitate visualization. (b) Critical buckling modes predicted by numerical analysis for supported square lattices with $t / h=0.1,0.3$ and 0.5 . The buckling mode is found not to be affected by $t / h$. This confirms that buckling-induces pattern of supported cellular structures is dictated by the aspect ratio $l / h$. 

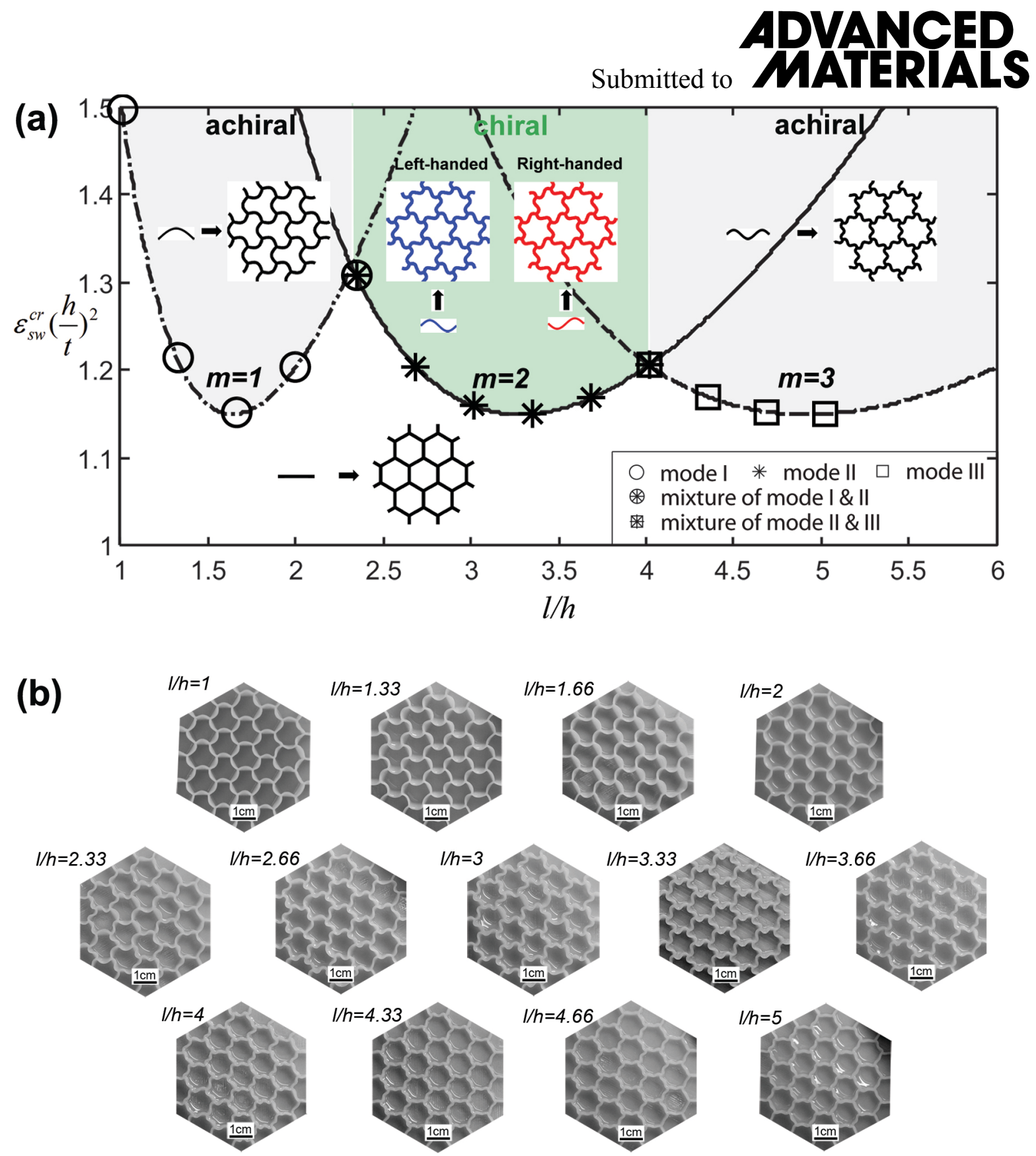

Supplementary Figure S3. Experimental verification of the stability diagram. (a) To verify the validity of our analytical model, we fabricated macroscale honeycomb structures $(l=5 \mathrm{~mm}$, $t=0.9 \mathrm{~mm}, h=1-5 \mathrm{~mm}$ ) made from silicone rubber with different aspect ratio $l / h$, as indicated by the markers in the stability diagram. (b) Buckling patterns observed in samples with different $l / h$. Modes characterized by $m=1, m=2$ and $m=3$ are observed, exactly as predicted by the analytical model. Moreover, mixtures of modes with $m=1-2$ and $m=2-3$ are found for geometries lying on the boundary of adjacent regimes (Figure S3(a)). 


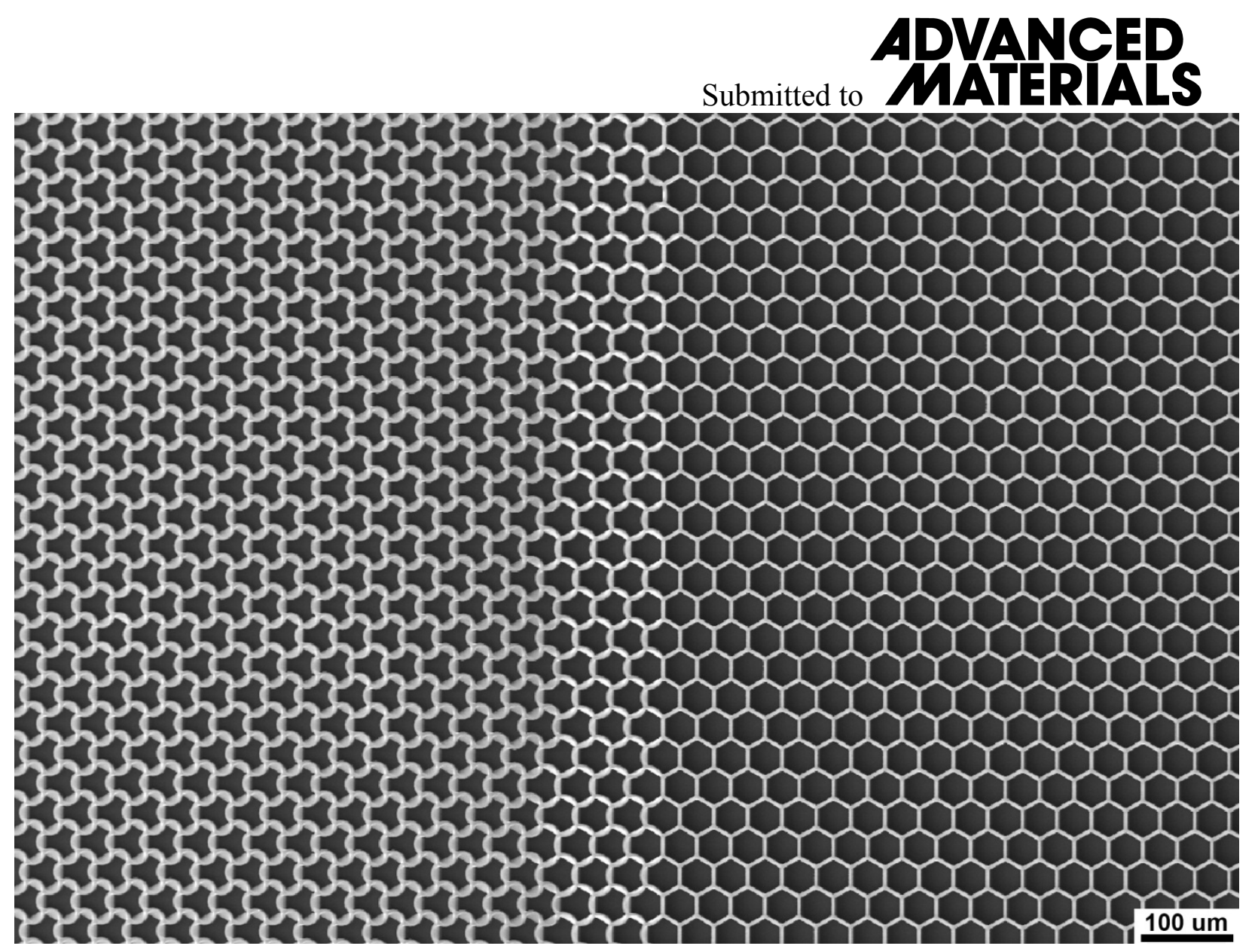

Supplementary Figure S4. Scanning electron microscope (SEM) image of buckled patterns

formed by compression of surface-attached honeycombs. Compression was applied to the left half of a micro-scale honeycomb structure $(l / h=2, t / h=0.37)$. The compressed region shows a uniform, $1^{\text {st }}$ mode buckled pattern. 